西

(1)

(2)

-

Revista Electrónica Enfermería Actual en Costa Rica

www.revenf.ucr.ac.cr

\title{
Programa educativo en salud sexual y salud reproductiva dirigido a la población femenina víctima del comercio sexual ${ }^{1}$
}

\author{
Karen Umaña Chacón ${ }^{2}$ \\ Karla Chaves Mayorga ${ }^{3}$
}

Institución: Fundación Rahab, Costa Rica

\section{CÓMO CITAR}

Umaña, K. y Chaves, K. (2012). Programa educativo en salud sexual y salud reproductiva dirigido a la población femenina víctima del comercio sexual. Rev. Enfermería Actual en Costa Rica, 23, 1-10 Recuperado de: http://www. revenf.ucr.ac.cr/saludsexual.pdf ISSN 1409-4568

\section{Resumen}

El objetivo de esta investigación fue desarrollar un programa educativo en Salud Sexual y Salud Reproductiva dirigido a la población femenina víctima del comercio sexual que asiste a la Fundación Rahab. Se partió de un enfoque cuantitativo para realizar un diagnóstico de necesidades educativas. La población participante fue de 20 mujeres, quienes están cursando una serie de módulos instructivos en Rahab. Se diseñó, ejecutó y evaluó el programa educativo, tomando en cuenta también las recomendaciones sugeridas del diagnóstico, psicólogas a cargo y profesionales de Enfermería. Se aplicaron 2 cuestionarios para recolectar los datos, la evaluación del programa se llevó a cabo por cada uno de los temas expuestos. De acuerdo con los resultados de la evaluación (pre y post-test), las participantes lograron alcanzar el objetivo educativo propuesto. Por otro lado, según las opiniones de las mujeres participantes, 16 de ellas calificaron al programa con una puntuación de cinco puntos de cinco y 20 mencionaron sentirse satisfechas con las actividades llevadas a cabo. Se concluye que el desarrollo de programas educativos sobre salud Sexual y reproductiva dirigidos a la población femenina víctima del comercio sexual, les permite empoderarse y acceder a diversas oportunidades sociales, así como maximizar sus funciones y calidad de vida personal y, por ende, la de sus hijos y familia en general.

Palabras clave: Comercio-sexual, Educación, Enfermería, Mujeres, Reproductiva, Salud-sexual.

\footnotetext{
${ }^{1}$ Fecha de recepción: julio 2012

Fecha de aceptación : agosto 2012

${ }^{2}$ Enfermera, Magister en Enfermería Ginecológica, Obstétrica y Perinatal. Correo Electrónico: karen1570@hotmail.com

${ }^{3}$ Enfermera, Magister en Enfermería Ginecológica, Obstétrica y Perinatal. Correo Electrónico: kachamay@costarricense.cr
} 


\section{Educational program on sexual and reproductive health for the female population victim of sex trade ${ }^{4}$}

Karen Umaña Chacón ${ }^{5}$ Karla Chaves Mayorga ${ }^{6}$

Institution: Fundación Rahab, Costa Rica

\section{CITED}

Zeledón, E. (2012) Educational program on sexual and reproductive health for the female population victim of sex trade. Rev. Enfermería Actual en Costa Rica, 23, 1-10. Available: http://www. revenf.ucr.ac.cr/ saludsexual.pdf ISSN 1409-4568

\section{ABSTRACT}

The objective of this research was to develop an educational program in Sexual and Reproductive Health for the female population of the sex trade victim attending the Rahab Foundation. We started with a quantitative approach for diagnosis of educational needs. The participant population was 20 women, who are attending a series of instructional modules in Rahab. We designed, implemented and evaluated the educational program, taking into account the recommendations of the diagnosis suggested, by psychologists and nurses. We applied two questionnaires to collect data, program evaluation was conducted for each of the topics. According to the results of the evaluation (pre and post-test), participants were able to reach the proposed educational goal. Furthermore, according to the views of women participants, 16 of them rated the program with a score of five out of five and 20 mentioned they were satisfied with the work carried out. We conclude that the development of educational programs on sexual and reproductive health aimed at the female population victim of the sex trade, and empower them to access various social opportunities and maximize their quality of life functions and personnel and hence that of his children and family in general.

Key words: Trade-sexual, Education, Nursing, Women, Reproductive, Health-sexual

\footnotetext{
${ }^{4}$ Fecha de recepción: julio 2012

Fecha de aceptación : agosto 2012

${ }^{5}$ Enfermera, Magister en Enfermería Ginecológica, Obstétrica y Perinatal. Correo Electrónico: karen1570@hotmail.com

${ }^{6}$ Enfermera, Magister en Enfermería Ginecológica, Obstétrica y Perinatal. Correo Electrónico: kachamay@costarricense.cr
} 


\section{INTRODUCCIÓN}

La prostitución es una de las ocupaciones más antiguas en el mundo; en la actualidad, es la segunda actividad industrial que genera más dinero, alrededor de $\$ 108.000$ millones anuales (Revista El Economista, 2010). Paralelamente a esta gran cantidad de dinero, existen países tradicionalmente emisores, como República Dominicana, Brasil, Colombia, y países receptores tales como Japón, Estados Unidos, España, Alemania e Israel (De Rosa, 2012), lo cual contribuye a la transmisión de diversas enfermedades y repercute, inevitablemente, en el bienestar de la salud pública.

La Organización Juventud Trinitario (2003) reportó un estimado mundial de 40 millones de personas en el ejercicio de la prostitución. En Costa Rica, el comercio sexual es un factor que atrae al turismo, como lo manifiestan Fernández y Rodríguez (2005) que "Costa Rica se encuentra al lado de Tailandia, Filipinas y otros destinos sexuales que constituyen a la ruta de visita de pedófilos, proxenetas y demás corruptos del llamado "primer mundo" (p. 38).

Por otro lado, Costa Rica es señalada, en Centroamérica, como país líder en promoción pornográfica y prostitución, el cual menciona:

Costa Rica como el país centroamericano con más páginas en la red que publicitaba la pornografía y la prostitución tanto femenina como masculina: Investigaciones oficiales determinaron que hay 40 páginas directas y aparecen 30 indirectas. (Carvallo y Rodríguez, 2005, s.p.)

Tardón (2012) se refiere al impacto del ejercicio del comercio sexual en la salud de la población y afirma que las mujeres que se dedican a la prostitución en países de mediano y bajo ingreso tienen un riesgo 14 veces mayor de ser contagiadas del virus VIH Sida, que el resto de la población femenina. De igual forma Shannon y Montaner (2012), de la Universidad British Columbia, aseguran que el 50\% de las personas dedicadas a la prostitución viven con VIH Sida. Adicionalmente, Matey (1998) se refiere al estudio efectuado por la Dra. Melissa Farley, psicóloga e investigadora del Kaiser Foundation Research Institute (California), en el cual se evidencian los trastornos psíquicos que afectan a las prostitutas; el 68\% de estas sufren de estrés postraumático.

Además, la salud sexual y la reproductiva se fundamentan en conceptos de suma riqueza en su contenido, por ejemplo, la Organización Mundial de la Salud (OMS, 2002) define:

Salud sexual es un estado de bienestar físico, emocional, mental y social relacionado con la sexualidad; no es meramente la ausencia de enfermedad, disfunción o debilidad. La salud sexual requiere un acercamiento positivo y respetuoso hacia la sexualidad y las relaciones sexuales, así como la posibilidad 
de obtener placer y experiencias sexuales seguras, libres de coerción, discriminación y violencia. Para que la salud sexual se logre y se mantenga los derechos sexuales de todas las personas deben ser respetados, protegidos y cumplidos. (p. 5)

Por otro lado, la salud reproductiva fue definida por la Organización de Naciones Unidas (1994) como (...) un estado de completo bienestar físico, mental y el bienestar social en todos los asuntos relacionados con el sistema reproductivo y sus funciones y procesos. Esto implica que las personas tienen la capacidad de reproducirse y la libertad de decidir si, cuándo y con qué frecuencia debe hacerlo. Queda implícito en ello el derecho de los hombres y las mujeres a estar informados ya tener acceso a métodos seguros, eficaces, asequibles y aceptables de planificación de la familia de su elección, así como otros métodos de su elección para la regulación de la fecundidad, que no están en contra de la ley y el derecho de acceso a los servicios de salud que permitan a las mujeres para ir de forma segura durante el embarazo y el parto. (p.37)

Contrariamente al idealismo de estas definiciones, la explotación sexual es un opio que violenta los derechos fundamentales de cualquier individuo, indistintamente del género y grupo etario, pues coarta de manera implícita la salud física, emocional y mental de aquellas personas que están involucradas e imposibilita el desarrollo del individuo en ambientes sanos y con calidad de vida. Al respecto, Bolaños (2003) menciona que

La explotación sexual mundial supone una crisis para los derechos humanos de las

mujeres y niñas violándose, entre otros, los siguientes derechos : libertad, igualdad, dignidad, seguridad, prohibición de esclavitud y servidumbre, prohibición de torturas y de tratos crueles, inhumanos o degradantes, recursos efectivo ante los tribunales que la ampare contra actos que violen sus derechos fundamentales, prohibición de injerencias en la vida privada y de familia ni de ataques a su honra o reputación, a circular libremente y elegir residencia, a salir de cualquier país y regresar al propio, libertad de 
opinión y de expresión, libre elección de trabajo con condiciones equitativas y

satisfactorias, al descanso y disfrute del tiempo libre y, en definitiva, a un nivel de vida

adecuado con el disfrute de sus derechos civiles y socioeconómicos. (p.15)

Por las razones anteriores, los programas de educación en salud sexual y salud reproductiva crean espacios aptos para la transmisión de la información y el conocimiento, mediante los cuales la persona puede decidir acerca de cómo mejorar o modificar sus conductas o acciones. Además, se ofrece la posibilidad de ejercer, implícitamente, el preciado derecho a la información, tal y como lo menciona la OMS: "Toda persona tiene derecho a recibir información sexual y a considerar que las relaciones sexuales sirven para el placer además de servir para la procreación.” (OMS, 1975, p. 26)

Debido a la problemática expuesta, surge el interés, por parte de egresadas de la maestría en Enfermería Ginecológica, Obstétrica y Perinatal de la Universidad de Costa Rica, por realizar una investigación aplicada que proporcione información necesaria sobre salud sexual y salud reproductiva a mujeres víctimas del comercio sexual que asisten a la Fundación Rahab. Por lo tanto, se estableció el objetivo de desarrollar un programa educativo dirigido a esa población.

\section{MATERIALES Y MÉTODOS}

Al momento de llevar a cabo un diagnóstico educativo, la población participante fue de 20 mujeres activas. Se fundamentó esta investigación en los lineamientos que propone el enfoque cuantitativo de tipo descriptivo y transversal. Los criterios establecidos para la selección de las participantes fueron los siguientes: que pertenezcan a la Fundación Rahab y que tuvieran anuencia a participar.

Se aplicaron dos cuestionarios: uno tuvo como objetivo realizar un diagnóstico de necesidades educativas, constó de un listado de 11 temas sobre salud sexual y salud reproductiva, el otro; caracterizar a la población participante. Este estaba compuesto por 24 ítems de respuesta cerrada.

La metodología se subdividió en fases. Primeramente, durante la coordinación, se llevó a cabo una serie de reuniones, con administrativas y psicólogas de la fundación, para establecer el contacto con el grupo de mujeres víctimas del comercio sexual. Luego, se desarrolló una fase diagnostica en la cual se aplicó dos cuestionarios autoadministrados, además de poner en práctica la técnica de observación para identificar la dinámica en la Fundación Rahab. Posteriormente, se diseñó y se implementó la propuesta educativa en Salud Sexual y Salud Reproductiva dirigida a las mujeres participantes. Este programa contó con objetivos, contenido teórico, actividades planeadas, recursos didácticos y humanos y los horarios. La programación se comentó con las participantes durante el primer día de la intervención. Finalmente, en la fase de evaluación, se evaluó el proceso educativo al finalizar cada sesión mediante la técnica de preguntas y respuestas. También, se efectuó una examinación teórica (pre y post-test) por cada tema; para esta, se consideró la opinión de las participantes acerca del desarrollo del programa en general. 


\section{Consideraciones éticas.}

Para esta sección, los aspectos más relevantes fueron el anonimato de las participantes, así como el consentimiento informado. Se les detalló que este trabajo se llevaba a cabo únicamente con fines académicos y que incentivaba el interés de las investigadoras por brindarles el apoyo informativo en lo que se refiere a los temas de Salud Sexual y Salud Reproductiva.

\section{RESULTADOS}

En cuanto a la caracterización de la población, se observó que las participantes en su mayoría, 10 de ellas, se encontraban en edades entre los 18 y 29 los años seguido por un grupo de siete individuos que se encontraban entre los 30 y los 39 años.

Con respecto a la procedencia, 17 de las mujeres son de San José. En la variable de escolaridad, se destacó que 14 de las participantes completaron la enseñanza escolar primaria; y tres de ellas, hasta el noveno año. En relación con estado civil, se evidenció lo siguiente:

$\begin{array}{cl}\text { Estado civil } & \text { No. de mujeres } \\ \text { Solteras } & 9 \\ \text { Casadas } & 4 \\ \text { Unión libre } & 4 \\ \text { Divorciadas } & 3\end{array}$

En cuanto al número de hijos, se destaca que siete participantes tienen un hijo y siete, dos hijos.

También se indagó sobre las prácticas no saludables y se evidenció que el consumo de tabaco es la práctica predominante en el grupo, seguida por el alcohol; tan solo una de ellas consume drogas ilícitas.

Con respecto del inicio de las relaciones sexuales, se apreció que 17 de las mujeres iniciaron sus relaciones coitales antes de los 18 años; solo tres de ellas iniciaron entre el rubro de los 18 a los 29 años. En cuanto a la cantidad de compañeros sexuales que han tenido, la mayoría (14) señaló que superaba los 4 individuos. En relación con métodos anticonceptivos, las pastillas y el condón continúan siendo los de mayor popularidad entre dicha población.

Referente a la realización de examen de Papanicolaou, se evidenció que 18 de las féminas sí se lo habían realizado; los resultados de 17 de ellas se encontraban dentro del parámetro de lo "normal", tan solo una anotó tener displasia moderada. En cuanto a la realización del examen de mamografía, los resultados indicaron que la mayoría (14) no se lo ha realizado, cinco de ellas se lo han realizado una vez; una en dos ocasiones. Referente al autoexamen de mamas, 11 de ellas respondieron que no lo realizan.

Respecto de la cantidad de embarazos, se observó que seis de las mujeres han tenido un embarazo, cuatro tuvieron dos y otras cuatro tuvieron tres embarazos. 11 de las mujeres no reportaron abortos, ocho mencionó haber tenido un aborto y solo una presentó dos abortos. Se cuantificó cinco cesáreas en toda la población. 
En lo referente a las necesidades educativas, 17 de las mujeres mencionaron su preferencia hacia el tema de la prevención del cáncer de cérvix y papiloma humano. Un segundo tema de interés, para 14 de ellas, fue el de las infecciones de transmisión sexual; finalmente, la temática del aborto llamó la atención de siete de las participantes.

En cuanto a la implementación del programa, es posible concluir que, de acuerdo con los resultados de la evaluación (pre y post-test), las involucradas lograron alcanzar el objetivo educativo propuesto en el programa. Además, las opiniones de las mujeres demostraron el éxito del programa; 16 de ellas lo calificaron con una puntuación alta (cinco puntos de cinco) y 20 de las participantes mencionaron sentirse satisfechas con las actividades llevadas a cabo, así como con las respuestas que brindaron las investigadoras a las inquietudes y dudas que surgieron durante el desarrollo del programa.

\section{DISCUSIÓN}

La Fundación Rahab, nombre de una trabajadora sexual mencionada en la Biblia, en Costa Rica, se dedica desde 1997 a la atención de mujeres que emergen de la feminización de la pobreza con el trabajo sexual como fuente de ingresos para su hogar. La fundación les ofrece atención grupal e individual según necesidades; además, les proporciona la oportunidad de sanar diferentes áreas personales y emocionales afectadas por la victimización en el ejercicio del comercio sexual.

Algunas estrategias que lleva a cabo la Fundación Rahab, según (1996) son:

sensibilización e información a la comunidad sobre comercio sexual, atención individual socioeducativa terapéutica y espiritual a la familia entera, desarrollo de actividades lúdicas y recreativas para menores, articulación de red de servicios sociales, restitución de los derechos de las personas, atención psicológica, socioeducativa, terapéutica y espiritual, implementación de talleres para concientizar la problemática de explotación sexual, así como facilitación de procesos de capacitación técnico-vocacional. (s.p.)

Respecto de los programas educativos, en el repertorio de recomendaciones prácticas de la OIT sobre el VIH/sida (2001) explica que: 
Cuando sea práctico y apropiado los programas deberían: incluir actividades que inciten a las personas a sopesar los riesgos que corren personalmente (como individuos y como miembros de un grupo) y a reducirlos mediante unas decisiones oportunas, la negociación, las técnicas de comunicación y los programas educación, prevención y asesoramiento. (OIT, 2001, pp.19-20)

Pese a la indudable importancia de los programas de educación para lograr la prevención de la Salud Sexual y Salud Reproductiva, lo cierto es que las políticas de salud nacionales carecen de programas específicos dirigidos a grupos poblacionales compuestos por trabajadoras sexuales; esto entorpece el abordaje exitoso de dicha materia. De acuerdo a criterios de la misma OMS sobre los programas de salud, se afirma que para la elección de métodos para problemas de salud sexuales, se deben de tomar en cuenta tanto el problema específico a abordar, como los aspectos culturales del mismo enfermo, por lo cual se menciona que:

En el tratamiento de los problemas sexuales, al igual que en los demás problemas de salud, no puede haber un criterio universalmente aplicable, la elección del método dependerá del problema que haya de abordarse, de los condicionamientos culturales del enfermo y de la formación y la competencia del terapeuta. (OMS, 1975, p. 28).

Otro aspecto de gran relevancia social que concierne a un programa de Salud Sexual y Salud Reproductiva en un grupo de féminas que ejerce el comercio sexual, radica en lograr influir positivamente en cada mujer, de tal forma que se estimule un cambio en la forma en la que se percibe a sí misma. Lo anterior repercute, necesariamente, en su salud integral, pues influye en su estilo de vida, su salud sexual, su salud reproductiva, y en el empoderamiento de sus derechos sexuales y reproductivos. Todo esto mejora la condición de vida de la mujer, la cual tiene consecuencias en la familia y la sociedad.

Sobre la caracterización de las mujeres en el programa, se puede destacar que la mayoría de las féminas son bastante jóvenes. Monge, Rojas, Morales y Ramírez (2009) refieren que, de las "categorías" de trabajadoras sexuales, las ambulantes (grupo uno), son de mayor edad, menor nivel educativo, menor clase y acceso económico.

Según lo anterior, las mujeres del programa pueden ser clasificadas dentro de otros dos grupos: el segundo englobaría a aquellas que laboran en salas de masajes o como saloneras. El tercer grupo correspondería a las VIP o trabajadoras independientes. Asímismo, según el departamento de jefatura de Delitos sexuales del Organismo de Investigación Judicial, los principales lugares del país donde se concentran dichas prácticas son el Área metropolitana, lo que justifica que la mayoría de la población sea de la zona de San José, Puntarenas y Limón. En una cantidad menos importante, dicha actividad se desarrolla en otras provincias. 
Otro aspecto relevante es la baja escolaridad que acompaña a este tipo de trabajadoras; como factor común, este limita el acceso a oportunidades dentro de la sociedad y las obliga a realizar trabajos no dignificantes. Lo anterior confirma los elementos básicos de la feminización de la pobreza, condición en que tanto la mujer como sus hijos tienen pocas o nulas posibilidades de surgir. Cabe mencionar que la Programa Naciones Unidas para el Desarrollo (PNUD) (1995) refiere que de 1700 millones de pobres el 70\% son mujeres, fenómeno marcado en países del Sur e industrializados. La cantidad de compañeros sexuales, según Monge et al (2009), depende de o se asocia con la categoría de trabajo sexual que desempeñe la fémina.

Es importante destacar que, dentro de esa población de riesgo, existe un marcado desconocimiento sobre Salud Sexual y la Salud Reproductiva, lo cual las conduce al descuido de esta y aumenta las estadísticas de enfermedades prevenibles. Esto resulta visible al notar la poca constancia y seguimiento en la realización de exámenes, como el Papanicolau, y en la escasa práctica de autoexamen de mamas. En cuanto al aborto, este se relaciona con una baja condición económica, una alimentación deficiente, una baja escolaridad y precarias medidas de autocuidado. Sin embargo, este fenómeno se asocia principalmente con una condición laboral antagónica a la gestación en la cual garantías, tales como la incapacidad y la adecuación laboral en caso de una amenaza de aborto, resultan elementos distantes de la realidad laboral de estas mujeres. Aunado a esto, el ejercicio del comercio sexual es potencialmente concomitante con la realización de muchos otros delitos.

El abarcar cada uno de los temas que se desarrollan en el programa educativo constituye un aspecto esencial en la promoción de la salud y la prevención de la enfermedad. La promoción de la salud, según la OMS, constituye un proceso político y social global que abarca no solamente las acciones dirigidas directamente a fortalecer las habilidades y capacidades de los individuos, sino también las dirigidas a modificar las condiciones sociales, ambientales y económicas, con el fin de mitigar su impacto en la salud pública e individual. La promoción de la salud es el proceso que permite a las personas incrementar su control sobre los determinantes de la salud y en consecuencia, mejorarla. La participación es esencial para sostener la acción en materia de promoción de la salud.

Un aspecto ligado a la promoción de la salud es el de la prevención, términos usados en ocasiones juntos. De la misma forma que el término de promoción de la salud, la prevención se puede visualizar desde tres diferentes niveles de atención, clasificados, según Martínez (2001) en primario, secundario y terciario.

Prevención primaria es la identificación y atenuación de factores de riesgo que pueden predisponer a un individuo a desarrollar una enfermedad, la prevención secundaria es la detección e intervención sobre un proceso mórbido durante su fase preclínica o asintomática. Mientras que la prevención terciaria es la intervención en la historia natural de una enfermedad establecida y sintomática para maximizar la función y calidad de vida del paciente. (p. 238) 


\section{Revista Electrónica Enfermería Actual en costa Rica}

La Enfermería Ginecológica, Obstétrica y Perinatal es una especialidad que, mediante el desarrollo de programas educativos y otras estrategias de intervención, pretende promover la Salud Sexual y la Salud Reproductiva; esto especialmente en aquellas poblaciones de riesgo que carecen en muchas ocasiones de apoyos que les permitan acceder a diversas oportunidades sociales para el mejoramiento de su calidad de vida. De igual forma, la especialidad busca intervenir en la prevención de enfermedades para que, como lo señala Martínez (2009) maximice las funciones y calidad de vida personal y por ende la de sus hijos y familia en general.

\section{CONCLUSIÓN}

El desarrollo de programas educativos sobre salud Sexual y Salud Rpeproductiva dirigidos a la población femenina víctima del comercio sexual les permite empoderarse y acceder a diversas oportunidades sociales, esto ayuda a mejorar la calidad de vida personal y familiar.

\section{REFERENCIAS}

Bolaños, A. (2003). La prostitución desde una perspectiva de los derechos humanos. Ponencia presentada en la Comisión

Mixta Congreso Senado. Derechos Humanos de Médicos del Mundo, España. Recuperado de: http//www.observatorioviolencia.org/.../DOC1166017160_Prostitucion

De Rosa, G. (2012). Prostitución Globalizada. Mirada Global. Recuperado de http://www.miradaglobal.com/index.php?option=com_content\&view=article\&id=867:prostitucionglobalizada\&catid=30: sociedad\&Itemid=34\&lang=es

Organización Mundial de la Salud (2002). Archivo de sexología. La definición de salud sexual. Recuperado de: http://www2.hu-berlin.de/sexology/ECS5/definicion_4.html

El Economista (31 mayo 2010). Industrias que más dinero mueven en el mundo. El Econimista.es. Recuperado de: http://listas.eleconomista.es/economia/240-las-industrias-que-ms-dinero-mueven-en-el-mundo

Fernández, R. y Rodríguez, J. (2005) Elementos históricos sobre la prostitución femenina en Costa Rica. El caso del Valle Central Noroccidental. Diálogos: Revista electrónica de Historia. Recuperado de: http://www.historia.fcs.ucr.ac.cr/articulos/2005/prost-sramon.htm\#_edn2

Organización de las Naciones Unidas. (setiembre de 1994) Informe de la Conferencia Internacional sobre la Población y el Desarrollo. Recuperado de www.unfpa.org/webdav/site/global/shared/.../2004/icpd_spa.pdf

Organización Mundial de la Salud. (1975). Instrucción y asistencia en cuestiones de sexualidad humana : Formación de profesionales de la salud. (Informe técnico No. 572).Lugar: editorial.

Juventud Trinitaria (2003). Prostitución: Las cifras de una nueva esclavitud. Recuperado de: http://www.juventudtrinitaria.org/prostitucion.htm

Martínez, A (2001). La prevención en la atención de salud de la persona adulta mayor. En Morales, F. Temas Prácticos en Geriatría y Gerontología, Tomo II, (pp. 43-51) San José, Costa Rica: EUNED. 
Matey, P. (1998). El Vietnan de cada día. El Mundo Salud y Medicina. Recuperado de: http://www.elmundo.es/salud/310/24NO120.html

Monge-Nájera, J., Rojas, R., Morales, R. y Ramírez, A. (2009). Trabajo sexual femenino en la ciudad de San José, Costa Rica: Un enfoque sociobiológico al iniciarse el siglo XXI. Cuadernos de Investigación UNED 1(1), 27-31

Morales, M., Sisa, L. y Ávila, M. (1996). He aprendido a vivir bajo el sol. San José, Costa Rica: editorial.

Programa de Naciones Unidas para el Desarrollo (PNUD) (1995). Informe sobre Desarrollo Humano. Recuperado de http://hdr.undp.org/en/media/hdr_1995_es_indice.pdf

Shannon, K.,, Montaner, J. (2012)The politics and policies of HIV prevention in sex work. The Lancet Infectious Diseases, 12(7), 500-502 doi:10.1016/S1473-3099(12)70065-8

Oficina Internacional del Trabajo (2001). Repertorio de recomendaciones prácticas de la OIT sobre el VIH/Sida y el mundo del trabajo. Recuperado de: http://www.ilo.org/wcmsp5/groups/public/@ed_protect/@protrav/@ilo_aids/documents/publication/wcms_113788.pdf

Tardón, L. (20 de abril de 2012). Infecciosas en países de desarrollo. Diario El mundo.Recuperado de : http://www.elmundo.es/el mundosalud/2012/03/14/hepatitissida/133 
